\title{
O Simulador Didático de Ventilação Mecânica como ferramenta de ensino no contexto da pandemia da COVID-19
}

\section{The Mechanical Ventilation Didactic Simulator as a teaching tool in the context of the COVID-19 pandemic}

\author{
1 Tatiana de Assis Girardi taty_assis@yahoo.com.br \\ ${ }^{1}$ Daniel Girardi \\ ${ }^{1}$ Jefferson Luiz Brum Marques \\ ${ }^{1}$ Flávia Del Castanhel
}

\section{RESUMO}

O novo coronavírus (SARS-CoV-2) causa a doença COVID-19, declaradamente uma pandemia que ataca principalmente os pulmões, podendo a ventilação mecânica invasiva ser necessária. Além da catástrofe global, a pandemia causou uma preocupação mundial, entre elas a interrupção de aulas e treinamentos presenciais. O objetivo desse estudo foi comparar e analisar o uso do Simulador Didático de Ventilação Mecânica (SDVM) como ferramenta auxiliar no processo de ensino-aprendizagem em ventilação mecânica invasiva (VMI) antes e durante a pandemia da COVID-19. Para a coleta de dados, foi utilizado um questionário online elaborado pelos autores. Foram obtidas e analisadas 1.186 respostas no período de $1^{\circ}$ de março a 24 de junho dos anos de 2019 e 2020. Como resultado, o perfil dos usuários do SDVM é composto em sua maioria, por estudantes (graduação, pós-graduação e residência), do curso de Fisioterapia da rede privada de ensino. Referente aos profissionais, observou-se que com a pandemia da COVID-19, houve um aumento de $240 \%$ de respostas obtidas por eles comparado com o mesmo período de 2019, sendo fisioterapeutas a maioria. Por se tratar de uma ferramenta gratuita, sem limite de acesso ou de tempo, em 2020 o SDVM foi acessado 8.482 uma variação de 296,5\% comparado aos 2.139 acessos no mesmo período de 2019. Esse aumento sugere que, por existir uma carência de profissionais com experiência e conhecimento suficientes para o manuseio da VMI, o SDVM tenha sido empregado como uma ferramenta para auxiliar cursos de aprimoramento, capacitação e até mesmo para a formação de novos profissionais.

Palavras-chave: COVID-19. Simulação. Ventilação Mecânica Invasiva. Ensino.

\section{ABSTRACT}

New coronavirus (SARS-CoV-2) causes COVID-19 disease, which is avowedly a pandemic that mainly attacks the lungs, and invasive mechanical ventilation may be necessary. In addition to the global calamity, the pandemic has caused a worldwide worry, among them the interruption of classes and face-to-face training. This study aimed to compare and analyze the use of SDVM as an assisting tool in the teaching-learning process in invasive mechanical ventilation (IMV) before and during the COVID-19 pandemic. For data collection, an online questionnaire developed by the authors was used. A total of 1,186 responses were obtained and analyzed from March 1 to June 24, 2019, and 2020. As a result, the profile of SDVM users is mostly composed of students (undergraduate, graduate, and residency) from the private education Physical therapy course. Concerning professionals, we observed that with the COVID-19 pandemic, there was an increase of $240 \%$ of responses received by them compared to the same period of 2019, being physiotherapists the majority. As it is a free tool, with no access or time limit, in 2020 the SDVM was accessed 8,482 a variation of 296.5\% compared to 2,139 accesses in the same period of 2019. This increase suggests that because there is a lack of professionals with enough experience and know-how to manage IMV, the SDVM has been used as a tool to assist in improvement courses, training, and even training new professionals. Keywords: COVID-19. Simulation. Invasive Mechanical Ventilation. Teaching. 


\section{INTRODUÇÃO}

A doença COVID-19 causada pelo novo coronavírus da Síndrome Respiratória Aguda Grave (SARS-CoV-2), é declaradamente uma pandemia global. Até o dia 27 de julho de 2020, causou no mundo, mais de 600 mil mortes e infectou mais de dezesseis milhões de pessoas (OPAS, 2020).

A COVID-19 ataca principalmente os pulmões, causando disfunção e falha respiratória, que são a maior ameaça aos pacientes e a principal causa de morte. Assim, o suporte ventilatório é fundamental para aliviar os sintomas e salvar vidas. Para 5\% das pessoas gravemente infectadas pelo novo coronavírus, a ventilação mecânica invasiva (VMI) pode ser necessária (YI, Y.; 2020).

Para o manuseio da VMI é requerido profissionais da saúde muito bem treinados e experientes com adequado conhecimento para esta função. Porém, existe uma carência por esses profissionais (MOULI et al., 2020; BRASIL, 2020a). Em uma pesquisa realizada com residentes de medicina norte-americanos, 45\% dos entrevistados relataram que raramente ou nunca sentiam confiança para o manejo de pacientes ventilados mecanicamente (BYARS; KLINKHAMMER; FELLIN, 2019). No Brasil, um estudo realizado para avaliar o conhecimento sobre ventilação mecânica de estudantes do último ano de medicina, residentes e médicos da emergência, a maioria (89\%) disse que não recebeu informação e treinamento suficientes para o manuseio de um paciente em ventilação mecânica. Entre os pesquisados, $69 \%$ afirmaram que teriam dificuldades para ligar um ventilador e 77\% disseram que temeriam pela segurança do paciente caso necessitassem iniciar a VMI (TALO et al., 2017). Portanto, o treinamento insuficiente pode ser uma das causas dessa carência por profissionais experientes e treinados em VMI (SCHROEDL et al., 2012; LINO et al., 2016).

A fim de contribuir no processo de ensino-aprendizagem e promover treinamento em VMI, foi desenvolvido em 2016, o Simulador Didático de Ventilação Mecânica (SDVM). De acordo com a Sociedade para Simulação em Saúde, "Simulação é a imitação ou representação de uma ação ou sistema por outro. Pode-se dizer que as simulações de saúde têm quatro objetivos principais: educação, avaliação, pesquisa e integração de sistemas de saúde para facilitar a segurança do paciente” (SSH, 2020).

O SDVM é um simulador baseado na Web para ser usado por acadêmicos e profissionais de saúde como uma ferramenta de estudo da VMI ou para ser adotado pelo professor em suas aulas, sejam presenciais ou remotas, como uma ponte educacional entre as aulas e a avaliação a beira do leito de um paciente crítico, associando a teoria com a prática. Por ser uma ferramenta Web, para ser utilizado basta ter um navegador de internet e acessar gratuitamente o SDVM pelo endereço eletrônico http://sdvm.ufsc.br (DE ASSIS GIRARDI; GIRARDI; MARQUES, 2020).

A pandemia da COVID-19 causou dentre várias outras coisas, mudanças no ensino. Foram necessárias medidas para garantir o distanciamento social, como a interrupção das aulas presenciais e a adoção das aulas remotas por meio do uso de tecnologias digitais de informação e comunicação (TDIC). Por mais de uma década, as TDIC já vinham sendo utilizadas como ferramentas facilitadoras para o processo de ensino-aprendizagem e para o treinamento na área da saúde. Mas, a situação atual promoveu e acelerou a adoção, pelos professores, de ferramentas como: vídeos, podcasts, realidade virtual simples, jogos educacionais digitais e simulações em computador (GOH; SANDARS, 2020; ROSE, 2020).

Diante desse cenário, o presente estudo teve o objetivo de comparar e analisar o uso do SDVM como ferramenta auxiliar no processo de ensino-aprendizagem em VMI antes e durante a pandemia da COVID-19, a partir da análise de um questionário. 


\section{MÉTODOS}

O projeto deste estudo foi submetido ao Comitê de Ética e Pesquisa em Seres Humanos da Universidade Federal de Santa Catarina (Florianópolis) sob o número CAAE: 00913518.9.0000.0121 e teve parecer favorável.

Estudo observacional do tipo transversal descritivo com abordagem qualitativa. O levantamento foi feito em um período de tempo preestabelecido e posteriormente foram descritas as variáveis, seus padrões de distribuição e características da amostra estudada.

A partir de uma amostragem não probabilística, participaram deste estudo todos os usuários que acessaram o site do SDVM e que, de forma voluntária, responderam um questionário. Para este estudo, foram analisados os números de acessos no site do SDVM e as respostas dos usuários no questionário. Os dados foram obtidos entre $1^{\circ}$ de março a 24 de junho dos anos de 2019 e 2020. Foram excluídas respostas repetidas que o sistema computou com até um minuto de diferença entre elas. Isso pode ocorrer, quando o usuário clica mais de uma vez no botão enviar no formulário.

Para a coleta de dados, utilizou-se um questionário estruturado construído pelos pesquisadores via formulário Google. Portanto, o questionário é online (o link para acesso do questionário está no próprio site do SDVM), autoaplicado e individual realizado em um único momento. O questionário foi dividido em: estudantes (graduação, pós-graduação e residência) e profissionais. A partir da resposta da primeira pergunta, o usuário já era direcionado às perguntas correspondentes ao perfil a que ele pertencia (estudante ou profissional). O questionário para estudantes continha cinco questões fechadas. Já o questionário para profissionais incluía três questões fechadas. É importante mencionar que para responder o questionário, o usuário não precisava de identificação e todos os dados obtidos foram tratados de forma totalmente anônima.

As variáveis utilizadas neste estudo são categóricas e seus valores foram armazenados em planilhas do Microsoft Excel e posteriormente analisados, com o uso da linguagem R versão 4.01 (R CORE TEAM, 2018), sendo calculadas as frequências absolutas e percentuais. Para verificar possíveis associações entre variáveis foi empregado o teste qui-quadrado $\left({ }^{2}\right.$ ) de independência com nível de significância estabelecido em 0,05 e empregado o ajuste de Bonferroni para identificar diferenças (DUNN, 1961; FRANE, 2015).

\section{RESULTADOS}

Para usar o SDVM, não é obrigatório responder o questionário. Portanto, o número de respostas do questionário não corresponde ao número de acessos no SDVM. O SDVM pode ser acessado sem a necessidade do usuário registrar-se e o mesmo usuário pode acessar sempre pelo mesmo dispositivo ou por dispositivos diferentes, sem limite de tempo ou de vezes em sua versão completa e gratuita. Por isso, não são obtidos os números de acessos dos usuários individualmente e sim, quantas vezes o simulador foi acessado, mesmo que tenha sido por um único usuário. No período estabelecido por esta pesquisa, foi observado que, em 2020, durante a pandemia da COVID-19, houve 8482 acessos, um aumento de 296,5\%, quando comparado aos 2139 acessos no mesmo período de 2019 (Figura 1). 
Figura 1- Gráfico do número de acessos no período de $1^{\circ}$ de março - 24 de junho dos anos de 2019 e 2020 no site do SDVM.

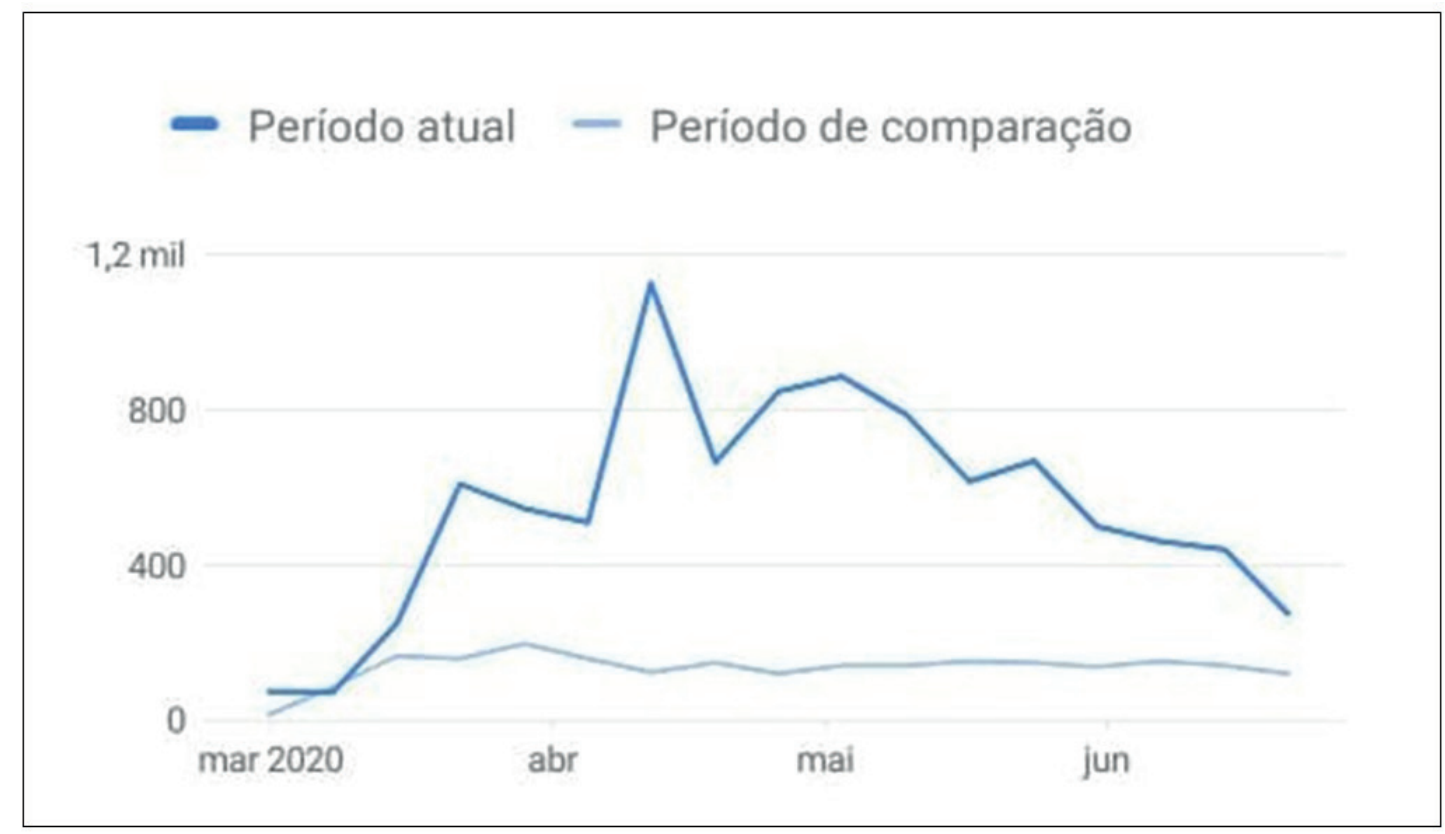

Fonte: Relatório do Google Analytics.

Dentro do período estabelecido foram consideradas 1.186 respostas ao questionário, as quais 424 (35,75\%) respostas foram obtidas em 2019 e 762 (64,24\%) em 2020. O fluxograma, disponível na Figura 2, sumariza esta seleção.

Figura 2 - Fluxograma das respostas incluídas.

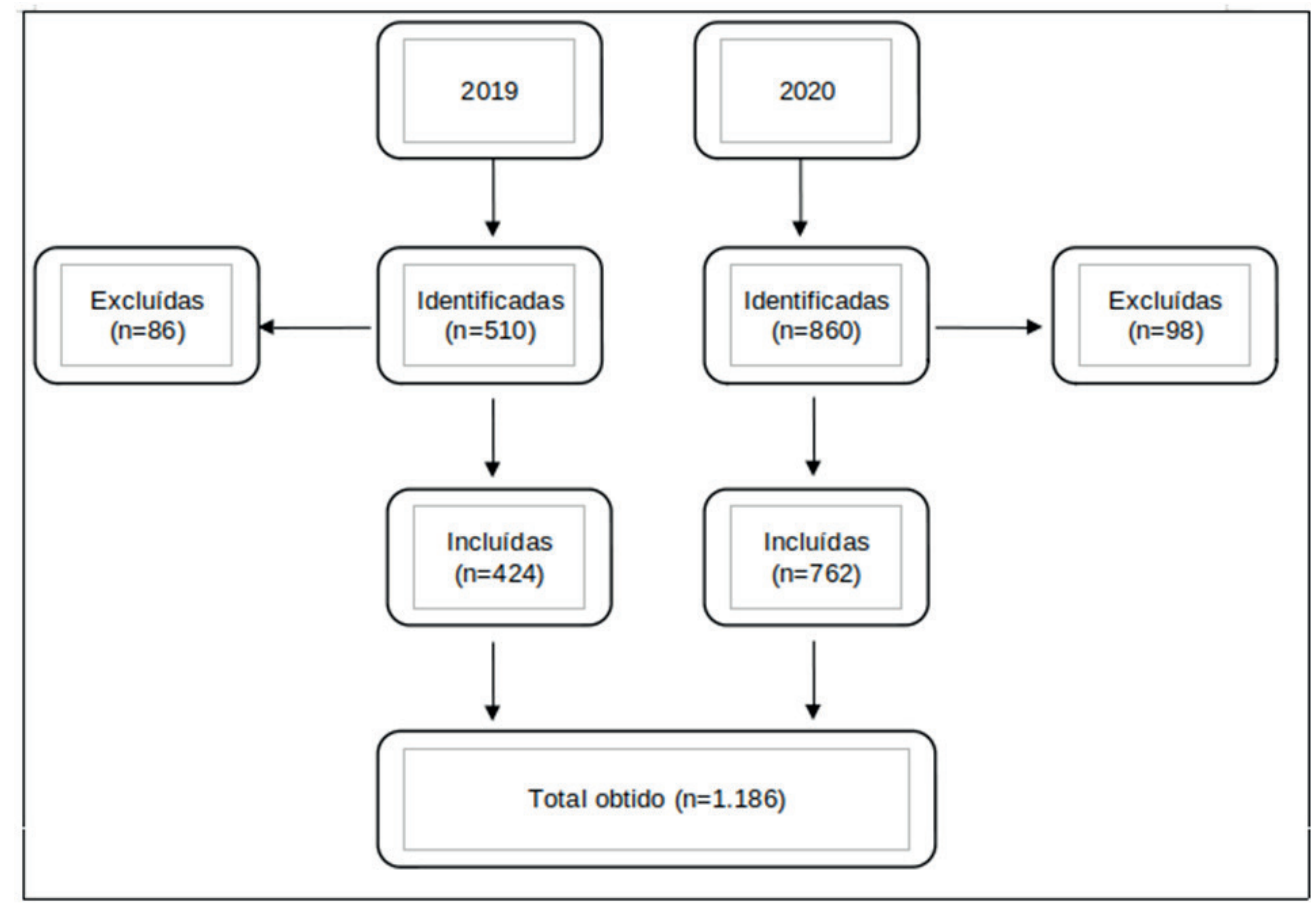

Fonte: Dos autores, 2020. 
Baseado nessas respostas, foi possível realizar um levantamento do perfil do usuário do SDVM. Foi observado que, das 424 (35,7\%) respostas obtidas no questionário, 345 (81,3\%) foram de estudantes, ao passo que 79 (18,6\%) de profissionais. Em 2020, durante a pandemia, apesar de 482 (64,2\%) respostas terem sido fornecidas por estudantes, 269 (35,8\%) respostas foram dadas por profissionais, observando um aumento de $240 \%$ de respostas por esse grupo de usuários (Figura 3).

Figura 3 - Prevalência do perfil dos usuários do SDVM no período de $1^{\circ}$ de março - 24 de junho de 2019 e de 2020 (n=1186).

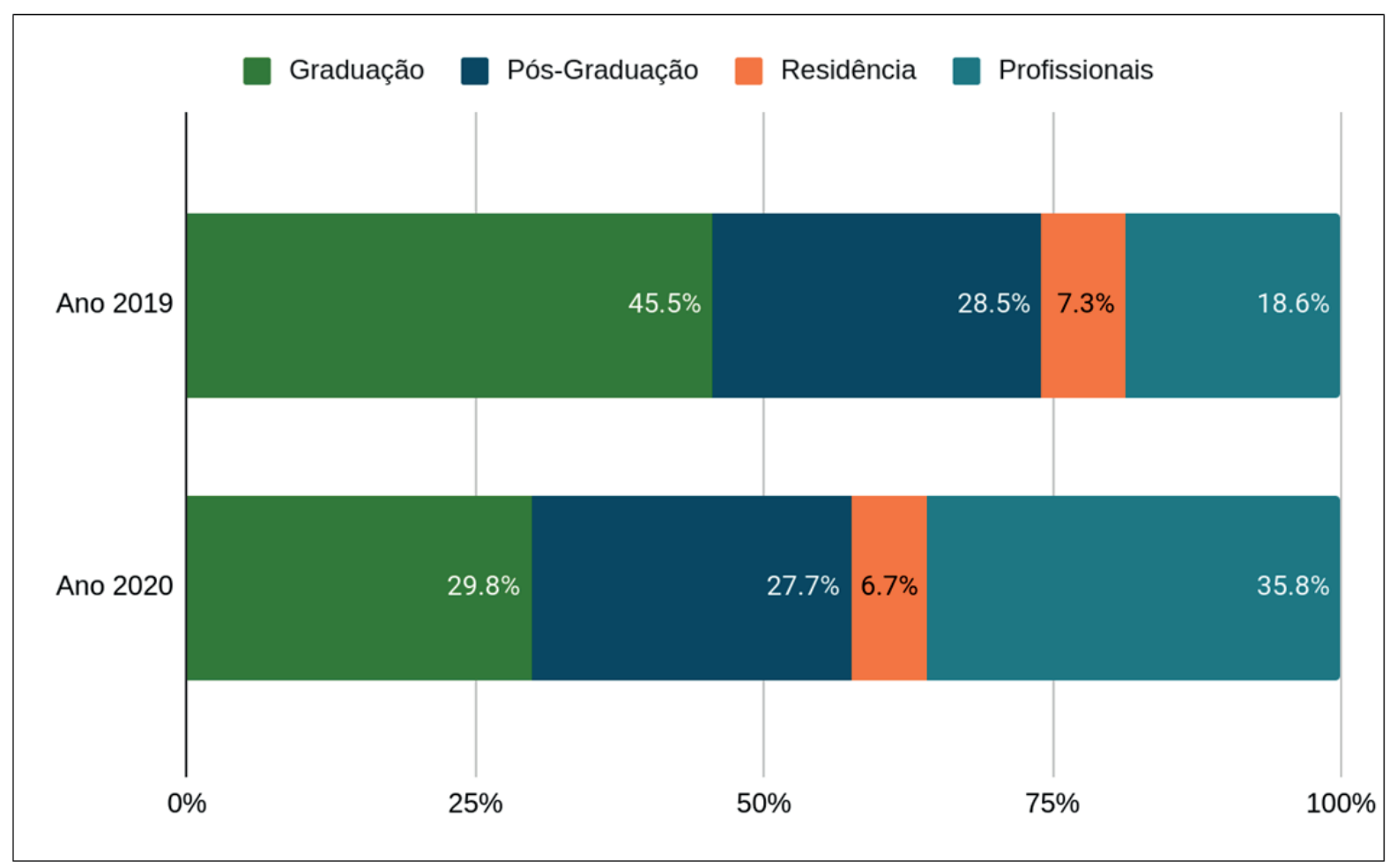

Fonte:Dos autores, 2020.

O questionário também permitiu realizar um levantamento do curso que os estudantes de graduação estavam cursando ou o curso que os estudantes de pós-graduação ou de residência haviam cursado na graduação. Ainda a respeito dos estudantes, foi possível identificar se a Instituição de Ensino Superior (IES) dos estudantes de graduação e de pós-graduação era pública ou privada. Para os residentes, também foi questionado se o Hospital em que estavam realizando a residência era da rede pública ou privada. A fim de facilitar a visualização, os residentes ao responderem a rede hospitalar, foi somado ao tipo de IES. Com relação aos profissionais, foi possível identificar a categoria profissional, sendo os Fisioterapeutas os profissionais que mais responderam o questionário no período dos dois anos analisados. Por fim, foi questionado como o usuário encontrou o SDVM e a pesquisa na Internet obteve a maioria das respostas nos dois anos (Tabela 1). 
Tabela 1 - Respostas obtidas no questionário do SDVM no período de $1^{\circ}$ de março a 24 de junho de 2019 e de $2020(n=1186)$.

\begin{tabular}{|c|c|c|}
\hline & $\begin{array}{c}\text { Ano } 2019 \\
\text { n (\%) }\end{array}$ & $\begin{array}{c}\text { Ano } 2020 \\
\text { n (\%) }\end{array}$ \\
\hline \multicolumn{3}{|l|}{ Variáveis } \\
\hline \multicolumn{3}{|l|}{ Curso (cursando ou cursado) } \\
\hline Enfermagem & $46(13,3)$ & $53(11,0)$ \\
\hline Fisioterapia & $232(67,2)$ & $302(62,7)$ \\
\hline Medicina & $62(18,0)$ & $111(23,0)$ \\
\hline Outros & $5(1,4)$ & $16(3,3)$ \\
\hline \multicolumn{3}{|l|}{ Instituição } \\
\hline Púbica & $72(20,9)$ & $131(27,2)$ \\
\hline Privada & $273(79,1)$ & $351(72,8)$ \\
\hline \multicolumn{3}{|l|}{ Profissionais } \\
\hline Enfermeiro & $13(16,5)$ & $38(14,1)$ \\
\hline Médico & $45(57,0)$ & $147(54,6)$ \\
\hline Técnico em enfermagem & $17(21,5)$ & $70(26,0)$ \\
\hline Outros & $3(3,8)$ & $10(3,7)$ \\
\hline \multicolumn{3}{|l|}{ Como encontrou o SDVM } \\
\hline Pesquisa na internet & $282(66,5)$ & $452(60,2)$ \\
\hline Redes socais (Facebook, Instagram) & $12(2,8)$ & $92(12,3)$ \\
\hline Indicação do(a) professor(a) & $106(25,0)$ & $116(15,4)$ \\
\hline Indicação de amigo & $24(5,7)$ & $91(12,1)$ \\
\hline
\end{tabular}

Nota: n (número); \% (porcentagem)

Fonte: Dos autores, 2020.

Ao comparar o nível o qual os estudantes pertenciam, observamos uma desproporção quando os comparamos com acessos ao SDVM. Portanto, o nível de significância de $\mathrm{p}=0,05$ após correção de Bonferroni foi ajustado para $\mathrm{p}=0,017$ para identificar essas diferenças. 
Entre os participantes que responderam ao questionário, houve a prevalência dos acessos ao SDVM por estudantes de graduação ( $p=0,007)$ em relação aos de pós-graduação $(p=0,021)$ e de residência $(p=0,483)$.

Ainda em relação aos estudantes, não foi observado prevalência de acessos por curso $(p=0,78)$ e a rede da IES ou do hospital, que o estudante pertencia $(\mathrm{p}=0,77)$. Igualmente, não foi identificado predomínio de acessos no SDVM por nenhuma das categorias profissionais envolvidas neste estudo $(\mathrm{p}=0,93)$.

\section{DISCUSSÃO}

A doença COVID-19 possui uma letalidade por volta de 5\%, mas apresenta alto grau de contaminação devido à velocidade com que se propaga e contamina as pessoas (ROTHAN; BYRAREDDY, 2020). Por isso, medidas de isolamento social tiveram que ser adotadas, o que causou impacto e transformações rápidas no âmbito social, econômico e educacional em vários países do mundo.

No contexto da educação superior no Brasil, o semestre que estava em andamento, precisou ser interrompido de maneira presencial e novas estratégias para o ensino tiveram que ser tomadas. Portanto, em março de 2020, foi publicada a Portaria n ${ }^{\circ} 343$ do Ministério da Educação (MEC), que: Dispõe sobre a substituição das aulas presenciais por aulas em meios digitais enquanto durar a situação de pandemia do Novo Coronavírus COVID-19.

Com a necessidade de adotar as TDIC para realizar as aulas remotas, iniciou-se o aumento na procura por essas ferramentas, sendo a simulação uma delas. Isso pôde ser observado na quantidade de acessos no site do SDVM e de respostas que foram obtidas no questionário. Na Figura 1 é possível visualizar a comparação entre o número de acessos nos dois anos ao longo do período da pesquisa. Observou-se que o aumento nos acessos ocorreram em meados de março, com o pico de acessos em abril coincidindo com o registro da primeira morte por COVID-19 no Brasil e as medidas de isolamento social. Com relação às repostas ao questionário no período de $1^{\circ}$ de março a 24 de junho em 2019, foram obtidas 424 respostas, enquanto no mesmo período em 2020, foram 762 respostas, o que corresponde a um aumento de quase 80\% de respostas em 2020.

Em relação a prevalência do perfil de usuários do SDVM que responderam ao questionário foi observado que, apesar da maioria das respostas ainda serem de estudantes, as respostas dos profissionais em 2020, teve um aumento de $240 \%$ comparado com o mesmo período analisado do ano anterior.

Esse aumento das respostas no questionário por profissionais pode ser reflexo de três possíveis situações. A primeira é que, as formas graves da COVID-19, podem demandar a ocupação de um leito em uma Unidade de Terapia Intensiva (UTI) de duas a quatro semanas. Esse tempo causa uma limitação estrutural por falta de leitos e de ventiladores mecânicos, o que exigiu que fossem criados mais leitos e adquiridos mais ventiladores mecânicos (HOLANDA; PINHEIRO, 2020). Porém, segundo o Ministério da Saúde (MS), há também uma escassez de profissionais especializados em VMI (BRASIL, 2020a). Além disso, muitos profissionais da linha de frente, por contraírem COVID-19, precisam ser afastados, agravando ainda mais a falta dessa força de trabalho especializada. Por isso, profissionais sem experiência e conhecimento necessários para a assistência desses pacientes, têm sido recrutados para atuarem. Em abril de 2020, O MS criou uma Ação Estratégica chamada “O Brasil Conta Comigo - Profissionais da Saúde”, com o objetivo de cadastrar profissionais de diferentes áreas da saúde para reforçar no enfrentamento ao novo coronavírus (BRASIL, 2020c).

A escassez de mão de obra qualificada e o recrutamento de novos profissionais pode ser a segunda situação que gerou o aumento do número de respostas no questionário por profissionais. Com a falta de conhecimento e prática, os profissionais inexperientes procuraram por ferramentas e cursos que auxiliassem na compreensão e no manuseio de ventiladores mecânicos. Para atender essa busca, foi observado um aumento de cursos online. Em maio de 2020, foi formalizada uma parceria entre o SDVM e o Conselho Federal de Fisioterapia e Terapia 
Ocupacional (COFFITO) para disponibilizar o uso do simulador durante um curso online e dar treinamento aos fisioterapeutas envolvidos no enfrentamento da crise provocada pela Pandemia da COVID-19, uma vez que, esses equipamentos são complexos e o inadequado manuseio pode gerar graves iatrogenias e inclusive, a morte do paciente.

A terceira situação foi a de que, como as aulas práticas e em laboratórios foram suspensas, por meio da Portaria $\mathrm{n}^{0} 345$ do MEC (BRASIL, 2020c), professores (profissionais) tiveram que buscar por ferramentas que permitissem simular o manuseio de um ventilador mecânico e adotá-las em suas aulas remotas. Sendo simulação uma estratégia utilizada para aquisição e treinamento de habilidades (MOULI et al., 2020), alguns estudos (YEE et al., 2016; CIULLO et al., 2018), mostraram que essa estratégia aumentou significativamente a confiança, o conhecimento e a performance dos estudantes para manejar o paciente ventilado mecanicamente.

O uso de simuladores virtuais, como o SDVM, tem crescido, principalmente durante o processo de ensino-aprendizagem de assuntos complexos, como a VMI, porque esses simuladores replicam não só o layout do aparelho, mas também o seu funcionamento, ao permitirem a configuração e a observação dessa configuração em um ambiente seguro e controlado. Neste cenário da Pandemia em que professores precisam realizar aulas síncronas e assíncronas, o uso de simuladores virtuais de VMI são importantes ferramentas, pois permitem que os professores, de forma interativa, simulem diferentes situações clínicas e o passo a passo para configurar o ventilador mecânico. Esse tipo de estratégia também gera reflexões e discussões ao serem resolvidos os casos clínicos propostos pelos professores, associando a teoria com a prática e a participação ativa do estudante no próprio processo de aprendizagem (DE ASSIS GIRARDI; GIRARDI; MARQUES, 2020). Para Mouli et al. (2020), o uso da simulação foi a melhor alternativa e foi efetivo no treinamento de residentes de medicina inexperientes para o manuseio da VMI na Pandemia da COVID-19.

Ainda sobre os profissionais, a maioria que respondeu ao questionário nos dois anos pesquisados, eram fisioterapeutas. De acordo com o estudo de Rotta et al. (2018), a assistência fisioterapêutica de 24 horas a pacientes de UTI promove menores custos hospitalares (custos totais e de pessoal) e menores comprometimentos motores e cardiopulmonares nos pacientes. Assim, mesmo a responsabilidade no manejo dos ventiladores mecânicos ser compartilhada entre as equipes de Enfermagem, Fisioterapia e Medicina, devido a esses resultados favoráveis das intervenções fisioterapêuticas, os fisioterapeutas conquistaram visibilidade, credibilidade e a autonomia no manuseio do ventilador mecânico (FU, 2018). Portanto, em tempos de Pandemia, os fisioterapeutas especialistas em Fisioterapia Cardiorrespiratória e em Terapia Intensiva têm sido considerados importantes integrantes da equipe multidisciplinar e vêm se destacando na linha de frente, por terem o conhecimento necessário para o manuseio dos ventiladores mecânicos.

De acordo com o levantamento realizado pela empresa de recrutamento online Catho, no período de março a maio desse ano, os cargos que apresentaram o maior aumento de vagas comparados ao mesmo período do ano anterior, foi em primeiro lugar, o de fisioterapeuta respiratório, com um aumento de $4.480 \%$, seguido pelo fisioterapeuta hospitalar, com um aumento de 1.555\% (CREFITO 5, 2020).

Assim, quanto ao perfil dos estudantes que responderam ao questionário, a maioria desses estudantes estavam cursando ou haviam cursado Fisioterapia, sendo isso, o reflexo da emancipação dos fisioterapeutas como integrante na equipe multidisciplinar das UTIs e o ganho de independência para gerenciar a VMI. Toda essa mudança repercutiu no processo de ensino-aprendizagem dos conteúdos de VMI nos cursos de Fisioterapia e na procura, tanto dos profissionais quanto dos estudantes, por ferramentas que contribuam nesse processo.

Quanto ao meio que os pesquisados utilizaram para encontrar o SDVM, a maioria nos dois anos, disse que o encontrou através de busca na Internet. Seguida pela busca na Internet, está a indicação dos professores. Vale ressaltar que, nas IES da rede privada, a substituição das aulas presencias por aulas remotas, devido à pandemia, ocorreu de maneira rápida. Desta forma, professores das universidades privadas tiveram que adaptar suas aulas para o novo formato e foram em busca das TDIC. Uma vez que, o professor usou nas aulas o SDVM, indicou-o 
aos seus alunos, os quais precisaram acessar o simulador para acompanhar as explicações, justificando também, o fato de ter mais respostas no questionário dos estudantes da rede privada de ensino do que da rede pública.

Este estudo apresentou limitações. A primeira, porque o questionário utilizado não é um questionário validado, o que pode gerar problemas na obtenção dos dados por simples erro de interpretação da pergunta. A segunda limitação também está relacionada com o questionário que apresentou apenas variáveis categóricas, o que dificultou fazer uma análise estatística mais robusta. E por último, como o sistema não tem controle de acesso por meio de registro individual de usuários, o número total de acessos leva em conta acessos repetidos realizados por um mesmo usuário, seja ele realizado em outro dispositivo ou em outra rede de Internet.

\section{CONCLUSÃO}

Os resultados apresentados por meio das respostas obtidas no questionário dentro do período estabelecido, revelaram o perfil dos usuários do SDVM, que é composto em sua maioria, por estudantes (graduação, pós-graduação e residentes), do curso de Fisioterapia da rede privada de Ensino Superior. Quanto aos profissionais que responderam ao questionário, o maior número corresponde aos fisioterapeutas. Em relação ao meio utilizado para encontrar o SDVM, a busca na Internet foi a maioria das respostas obtidas.

Apesar de ter sido observado que, tanto no ano de 2019 quanto no ano de 2020, o perfil do usuário do SDVM não mudou, em 2020 com a pandemia da COVID-19, houve um aumento de $240 \%$ de respostas obtidas por profissionais comparado com o mesmo período de 2019. Por se tratar de uma ferramenta gratuita, sem limite de acesso ou de tempo, em 2020, dentro do período analisado, o SDVM foi acessado 8.482 vezes, representando um aumento de 296,5\% comparado aos 2.139 acessos no mesmo período de 2019. Esses achados sugerem que, por existir uma carência de profissionais com experiência e conhecimento suficientes para o manuseio da VMI, observou-se a ampliação do uso do SDVM num contexto multidisciplinar, seja como uma ferramenta para auxiliar cursos de aprimoramento ou de capacitação dos profissionais, seja para a formação de novos profissionais.

\section{REFERÊNCIAS}

BRASIL. Ministério da Saúde (MS). Secretaria de Vigilância em Saúde. Boletim Epidemiológico número 8. Doença pelo coronavírus 2019. [Internet]. Brasília: MS; 2020a. Disponível em: < https://portalarquivos.saude. gov.br/images/pdf/2020/April/09/be-covid-08-final-2.pdf> Acesso em: 17 de jun. 2020.

BRASIL. Ministério da Educação (MEC). Portaria nº 343, de 17 de março de 2020. Dispõe sobre a substituição das aulas presenciais por aulas em meios digitais enquanto durar a situação de pandemia do Novo Coronavírus COVID-19. [Internet]. Brasília: Março de 2020b. Disponível em: <http://www.in.gov.br/en/web/dou/-/portarian-343-de-17-de-marco-de-2020-248564376>. Acesso em: 25 de jul. 2020.

BRASIL. Ministério da Saúde (MS). Secretaria de Vigilância em Saúde. “O Brasil Conta Comigo” cadastra 500 mil profissionais de saúde para atuarem no combate à Covid-19. [Internet]. Brasília: Abril de 2020c. Disponível em: $<$ https://www.gov.br/pt-br/noticias/saude-e-vigilancia-sanitaria/2020/04/201co-brasil-conta-comigo201d-cadastra500-mil-profissionais-de-saude-para-atuarem-no-combate-ao-coronavirus-pelo-brasil $\geq$ Acesso em: 26 de jul. 2020.

BRASIL. Ministério da Educação (MEC). Portaria no 345, de 19 de março de 2020. Altera a Portaria MEC $n^{\circ}$ 343, de 17 de março de 2020. [Internet]. Brasília: 2020d. Disponível em: <http://pesquisa.in.gov.br/imprensa/jsp/ visualiza/index.jsp?jornal=603\&pagina=1\&data=19/03/2020\&totalArquivos=1> Acesso em: 29 de jul. 2020. 
BYARS, D. V.; KLINKHAMMER, M. D.; FELLIN, M. A. In situ simulation for ventilator management in emergency medicine. AEM Education and Training. p. 1-4, 2019.

CIULLO, Anna et al. Telepresent mechanical ventilation training versus traditional instruction: a simulationbased pilot study. BMJ Simulation and Technology Enhanced Learning, v. 5, n. 1, p. 8-14, 2019.

CREFITO 5. Conselho Regional de Fisioterapia e Terapia Ocupacional 5. Fisioterapeuta respiratório e hospitalar são cargos com maior aumento de vagas durante a pandemia. [Internet]. 2020. Disponível em: <http://crefito5. org.br/noticia/fisioterapeuta-respiratorio-e-hospitalar-sao-cargos-com-maior-aumento-de-vagas-durante-apandemia>. Acesso em: 28 de jul. 2020.

DE ASSIS GIRARDI, T.; GIRARDI, D.; MARQUES, J. L. B. O uso de um simulador para o ensino de ventilação mecânica. Revista Brasileira de Informática na Educação, v. 28, p. 297-318, 2020.

DUNN, Ovive Jean. Multiple Comparisons among Means. Journal of the American Statistical Association, [S. l.], v. 56, n. 293, p. 52-64, 1961.

FIELD, Andy P.; MILES, Jeremy; FIELD, Zoë. Discovering statistics using R. SAGE Publications Ltd., 2012.

FRANE, Andrew V. Invited debate are per-family type I error rates relevant in social and behavioral science? Journal of Modern Applied Statistical Methods, [S. l.], v. 14, n. 1, p. 12-23, 2015.

FU, Carolina. Terapia intensiva: avances y actualizaciones en la actuación del fisioterapeuta. Fisioterapia e Pesquisa, v. 25, n. 3, p. 240-240, 2018.

GOH, P. S; SANDARS, J. A vision of the use of technology in medical education after the COVID-19 pandemic. MedEdPublish. p. 11-18, 2020.

HOLANDA, M. A.; PINHEIRO, B. V. Pandemia por COVID-19 e ventilação mecânica: enfrentando o presente, desenhando o futuro. Jornal Brasileiro de Pneumologia, v. 46, n. 4, p. e20200282-e20200282, 2020.

LINO, J. A.; et al. A critical review of mechanical ventilation virtual si-mulators: Is it time to use them? JMIR Medical Education. v. 2, n. 1, p.1-11, 2016.

MOULI, T.C.; et al. Effectiveness of simulation based teaching of ventilatory management among nonanaesthesiology residents to manage COVID 19 pandemic - A Quasi experimental cross sectional pilot study. Indian Journal of Anaesthesia. v. 64 sup 2, p. S136-S140, 2020.

OPAS. Organização Pan-Americana da Saúde. Folha Informativa - COVID 19 (doença causada pelo novo coronavírus) [Internet]. Brasília(DF); 2020. Disponível em: <https://www.paho.org/bra/index.php?option=com_ content\&view=article\&id=6101:covid19\&Itemid=875> Acesso em: 27 de jul.2020.

R CORE TEAM. A Language and Environment for Statistical ComputingR Foundation for Statistical Computing, 2018.

ROSE, S. Medical student education in the time of COVID-19. Jama. v. 323, n. 21, p.2131-2132, 2020.

ROTHAN, H. A.; BYRAREDDY, S. N. The epidemiology and pathogenesis of coronavirus disease (COVID-19) outbreak. Journal of Autoimmunity. v. 109, p. 1-4, 2020.

ROTTA, Bruna Peruzzo et al. Relationship between availability of physiotherapy services and ICU costs. Jornal Brasileiro de Pneumologia, v. 44, n. 3, p. 184-189, 2018. 
SCHROEDL, C. J.; et al. Use of simulation-based education to improve resident learning and patient care in the medical intensive care unit: a randomized trial. Journal of Critical Care. v. 27, n. 2, p. 219-e7-219.e13, 2012.

SSH. Society for Simulation in Healthcare. About simulation. [Internet]. Disponível em: <https://www.ssih.org/ About-SSH/About-Simulation> Acesso em: 17 de jun.2020.

TALLO, F. S.; et al. Evaluation of self-perception of mechanical ventilation knowledge among brazilian finalyear medical students, residents and emergency physicians. Clinics. v. 72, n. 2,p. 65-70, 2017.

YEE, Jennifer et al. Mechanical ventilation boot camp: a simulation-based pilot study. Critical care research and practice, v. 2016, p. 1-7, 2016.

YI, Y. et al. Covid-19: what has been learned andto be learned about the novel coronavirus disease. International Journal of Biological Sciences. v. 16, n. 10, p. 1753-1766, 2020. 